\title{
OTIOTOMTCS
}

Revista de economía, empresa y sociedad

ESTRATEGIAS PARA REDUCIR LA DESIGUALDAD

\section{Flexiseguridad o del paradigma del welfare al workfare en el actual contexto poscrisis español}

\section{Purificación Baldoví}

Profesora colaboradora de los Estudios de Economía y Empresa (UOC) Vocal independiente experta en innovación (CES, Comunidad Valenciana)

RESUMEN Actualmente, la economía española es más productiva y, sin embargo, no ha recuperado las tasas de empleo antes de la crisis, por lo que el principal reto es reducir el desempleo y el empleo temporal, crear empleos de calidad, estables y productivos, que ayuden a reducir la desigualdad y reanudar el camino de convergencia con las economías más avanzadas. El modelo de flexiseguridad define como una estrategia de modernización del mercado de trabajo a través de dos vías convergentes: flexibilidad (tanto empresarial como laboral, para responder a las necesidades de ambos) y seguridad (para los trabajadores, que deben poder desarrollar su carrera, sus habilidades y recibir apoyo de los sistemas de seguridad social durante períodos de inactividad). Sin embargo, este modelo de flexiguridad danesa no puede aplicarse directamente a cada Estado miembro o región de la Unión Europea, sino adaptarse a cada contexto con una combinación adecuada de instrumentos que respondan, en nuestra región, al debate entre diferentes sensibilidades sociales y políticas. Se busca la especialización flexible y no la flexibilidad precarizante.

PALABRAS CLAVE seguridad; flexibilidad; flexiseguridad; VUCA; economía colaborativa; digitalización; productividad; sostenibilidad; glocal 


\title{
Flexicurity or the paradigm of welfare to workfare in the current Spanish post-recession period
}

\begin{abstract}
The Spanish economy is more productive but employment rates have not returned to levels prior to the recession. Therefore, the main challenge is reducing unemployment and temporary employment, creating quality, stable and productive jobs that help reduce inequality, as well as resuming the path of convergence with more advanced economies. The model of flexicurity is defined as a strategy to modernize the labour market through two paths that converge: flexibility (for both business and workers, to respond to the needs of both) and safety (for workers who must be able to develop their careers, expand their skills and receive support from social security systems during periods of inactivity). However, this model of Danish flexicurity cannot be implemented directly to each Member State or region of the European Union, but rather it must be adapted to each context with an adequate combination of instruments that respond in our region to the debate between different social and political sensitivities. The goal is not increasing precariousness through flexibility, but rather flexible specialization.
\end{abstract}

KEYWORDS security; flexibility; flexicurity; VUCA; collaborative economy; digitalization; productivity; sustainability; glocal

\section{Introducción}

En un contexto VUCA (acrónimo que significa volatilidad, incertidumbre, complejidad y ambigüedad), las transformaciones que está experimentando el mundo del trabajo son estructurales y afectan al núcleo de las condiciones de producción y reproducción de la sociedad, definiendo nuevos horizontes para el empleo, y para todas las instituciones sociales principales: familia, escuela y gobierno.

En el contexto actual de España, tras destruir un 17,1 \% de la afiliación a la Seguridad Social (desde julio de 2007 a febrero de 2013), se ha recuperado ya el 68,3 \% del empleo destruido (junio 2017), es decir, que desde 2013 se ha producido un intenso proceso de creación de empleo, aunque la temporalidad sigue siendo elevada.

En definitiva, la economía española es más productiva pero no ha recuperado las tasas de empleo anteriores a la crisis por lo que el principal reto es reducir la tasa de paro y la temporalidad, creando empleo de calidad, estable y productivo, 
que ayude a reducir la desigualdad y a retomar la senda de convergencia con las economías más avanzadas.

Los interrogantes abiertos en esta nueva época están relacionados ahora con el ascenso de la «economía» colaborativa, el impacto de la digitalización en el trabajo y el tipo de demografía empresarial:

- En la llamada «economía colaborativa», existen nuevas vías de coordinación y de inclusión económica y financiera, y han emergido en sectores tradicionales como el alojamiento y el transporte (Airbnb, BlaBlaCar...).

- La digitalización supone una transformación disruptiva que genera un paradigma económico y laboral que aún no sabemos gestionar eficientemente. La digitalización transforma los puestos de trabajo, potencia la externalización y las deslocalizaciones (tareas, procesos y empresas), e incrementa la polarización y la exclusión de trabajadores con falta de competencias para abordar este nuevo escenario laboral, en el que las principales esferas del cambio tecnológico son la robotización, las máquinas inteligentes, el Big Data, la nube, la geolocalización, las plataformas digitales y el internet de las cosas.

- Por todo lo anterior, la demografía empresarial en el sector TIC aumenta, se consolida proyecta la aparición de nuevos productos y servicios, y provoca la mutación de las formas de organizar el trabajo y la producción.

Las cuestiones que subyacen en la reflexión sobre el mercado de trabajo actual giran en torno a sobre si está cambiando el concepto de «empleo», ya que las transformaciones anteriormente citadas suscitan interrogantes:

- ¿Se mantiene el concepto de empresario/a?

- ¿Está cambiando el tiempo de trabajo? ¿Hay un incremento del porcentaje de freelance/autoempleo o del multiempleo (empleos/tareas)?

- ¿Hay más autonomía, menos jerarquía en las organizaciones? ¿Es diferente la mentalidad de los jóvenes (millennials)?

- ¿Son escasos los datos de trabajadores en la economía colaborativa (1\%)?

- ¿Es la medición de la productividad mucho más sofisticada (privacidad)?

- ¿Continúa la externalización (outsourcing/offshoring) pero también la tendencia inversa?

- ¿Existe una competición global del talento?

- ¿Hay nuevas maneras de formarse, más informal, más a medida (MOOC)? 
Figura 1. Nuevo paradigma del mercado de trabajo

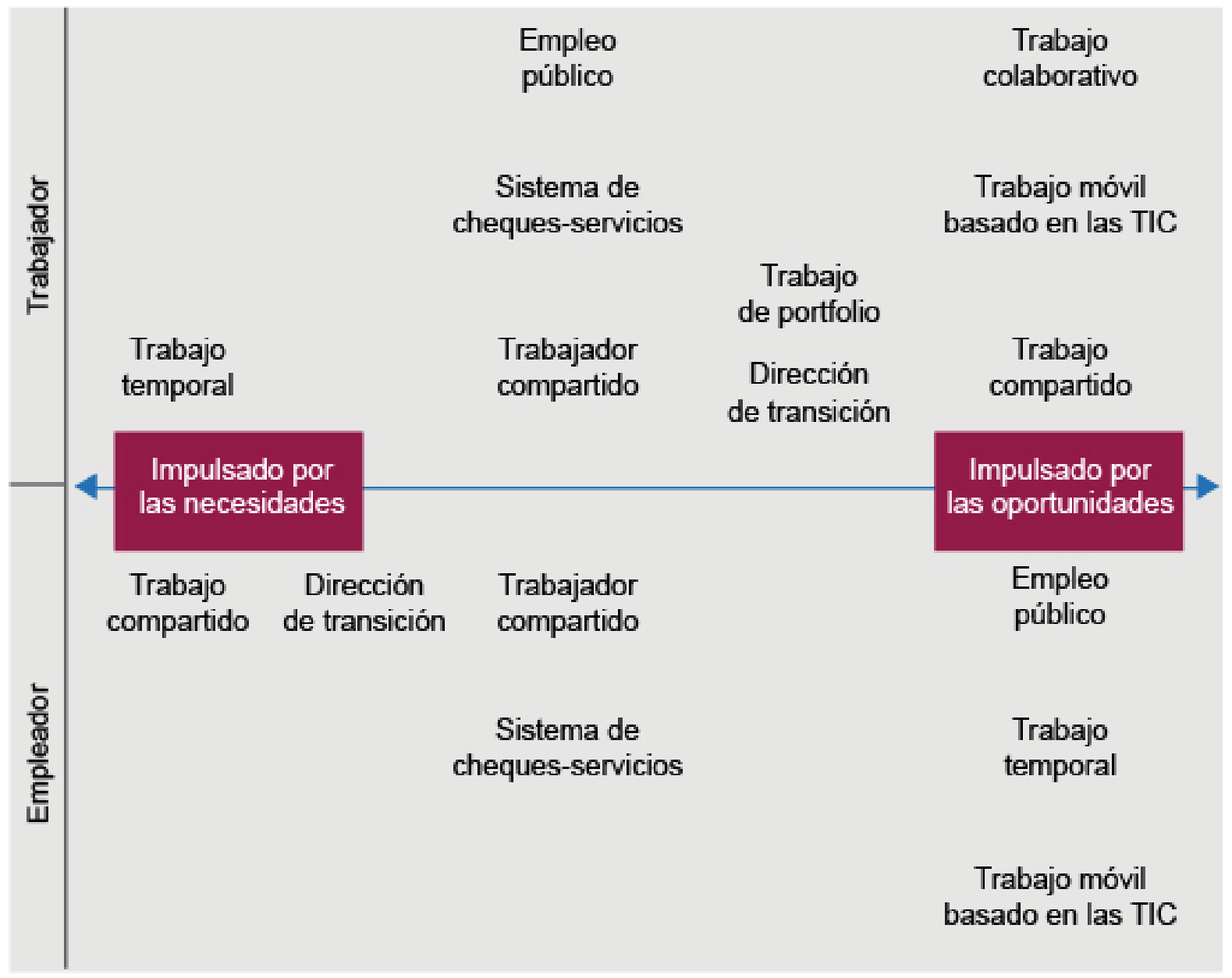

La respuesta al entorno es la de la flexiseguridad del mercado laboral, concepto que hace referencia al incremento de la flexibilidad (obtenido mediante facilidades en la contratación y en el despido) y un nivel alto de protección social (seguridad) a través de generosas prestaciones por desempleo, sujetas a la búsqueda proactiva de empleo y de formación continua.

\section{El modelo de flexiseguridad}

El modelo se define como una estrategia para modernizar el mercado laboral mediante dos vías que confluyen:

1. La flexibilidad, tanto empresarial como de los trabajadores, para responder a las necesidades de ambos. 
2. La seguridad para los trabajadores, que deben poder desarrollar sus carreras profesionales, ampliar sus competencias y recibir apoyo de los sistemas de seguridad social durante los períodos de inactividad.

La génesis de este modelo está asociada al modelo danés, que lo operativizó con éxito al combinar elevados subsidios por desempleo con bajos niveles de protección al despido, y suavizó así el desencuentro de la flexibilidad y la seguridad mediante un entorno suficientemente seguro con herramientas de protección y la compensación de la flexibilidad de los despidos con la seguridad de los trabajadores, que de este modo no entran en una espiral de paro y desfase formativo.

El énfasis en la protección o seguridad (con sus valores asociados: solidaridad, responsabilidad colectiva, derechos...) o en la activación o flexibilidad (o lo que es lo mismo: competencia, responsabilidad individual, exigencias...) pretenden confluir en una línea de encuentro entre las dos formas de entender la política laboral.

Sin embargo, este modelo de flexiseguridad danesa no puede ser implementado directamente en cada Estado miembro o región de la Unión Europea, sino que se ha de adaptar a cada contexto, con una adecuada combinación de instrumentos que den respuesta, en nuestra región, al debate entre distintas sensibilidades sociales y políticas.

No se busca la flexibilidad precarizadora, sino la especialización flexible.

\section{El equilibrio de intereses entre empresas y trabajadores}

El equilibrio de intereses entre empresas y trabajadores puede ser determinado mediante el diálogo social y las negociaciones con el Gobierno, de modo que existan garantías de seguridad para que trabajadores y empleadores inviertan en mejoras de la productividad (muy necesarias, especialmente en España) y la flexibilidad les permita adaptarse a la evolución del mercado y de la tecnología.

Esta transformación en la norma social de empleo ha de tender hacia la «flexibilidad sostenible» (Carnoy y Castells, 1997), de modo que se evite la «surafricanización» (Gorz, 1995) o de la «sudización del Norte» (Gallino, 2002) para que las características del llamado Tercer Mundo (trabajo precario, discontinuo e informal) no irrumpan en nuestra sociedad. 
Figura 2. Modelos de economía y sociedades capitalistas

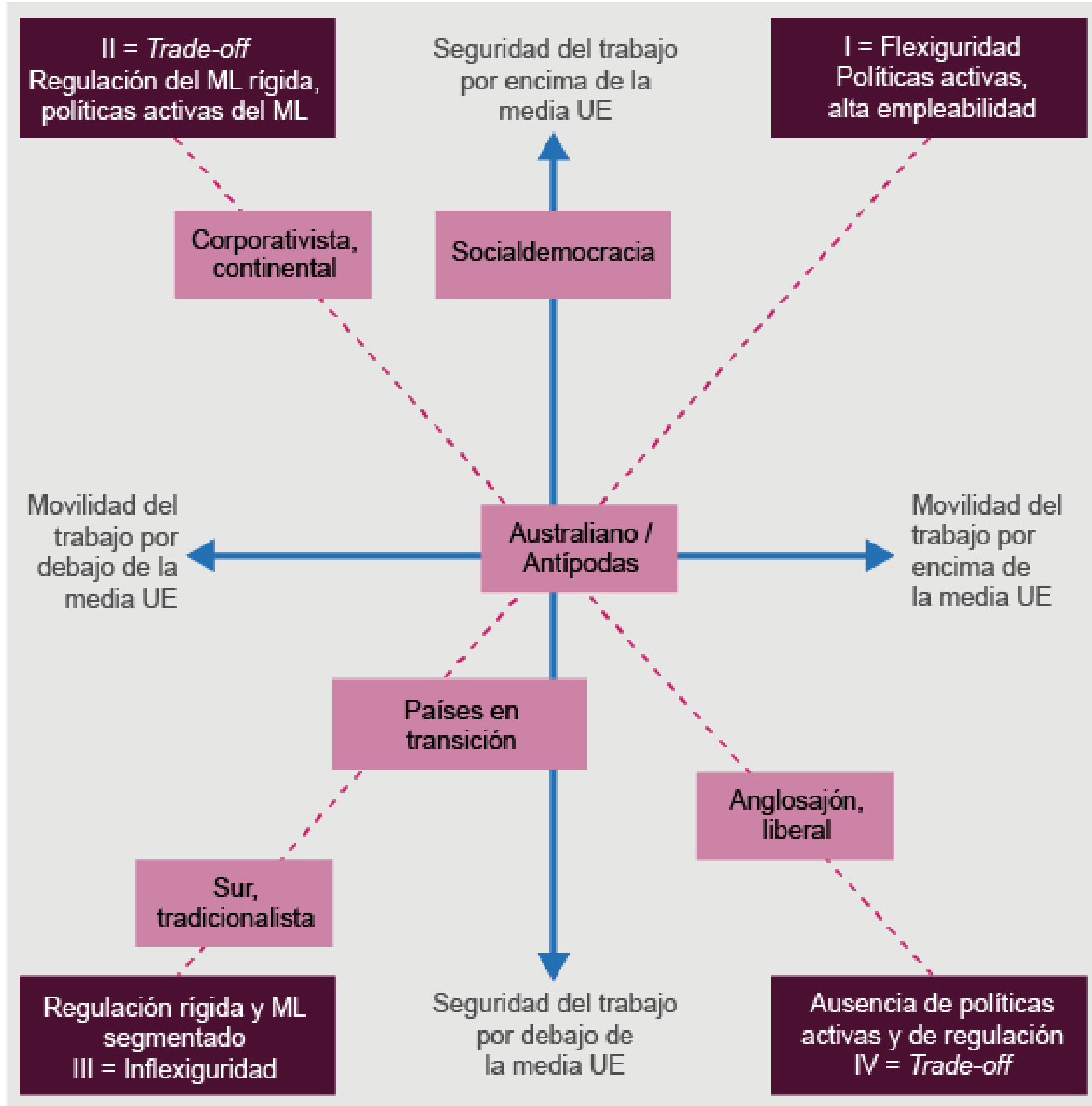

Fuente: Muffels y Wilthagen (2016).

Nota: Según Bruno Amable (2005), hay cinco capitalismos, cinco modelos de economías y de sociedades capitalistas: el modelo liberal (Australia, Canadá, Estados Unidos, Reino Unido), el modelo europeo continental (Alemania, Austria, Bélgica, Francia, Países Bajos, Irlanda, Noruega, Suiza), el modelo asiático (Corea del Sur, Japón), el modelo mediterráneo (España, Grecia, Italia, Portugal) y el modelo socialdemócrata (Dinamarca, Finlandia, Suecia). 


\section{Contexto español: baja productividad}

La baja productividad es un obstáculo para el crecimiento económico en nuestro país, lo cual es un freno para el desarrollo económico.

Según Emilio Ontiveros (2015), catedrático de Economía de la Empresa de la Universidad Autónoma de Madrid y fundador y presidente de Analistas Financieros Internacionales, las razones de la menor productividad española son:

1. La producción está concentrada mayormente en sectores manufactureros tradicionales.

2. Inferior crecimiento de la participación en la economía de las empresas del sector servicios en los que aumenta la productividad.

3. Menor contribución de las industrias productoras de TIC y de las empresas usuarias de las mismas al conjunto de la economía.

4. Reducidos incentivos empresariales para la asunción de riesgos.

5. Escasa complicidad del sistema educativo con la capacidad para emprender.

6. Reducida (o insuficiente) inversión en I+D.

\section{Orientaciones para avanzar en las soluciones}

Es imprescindible que la sociedad (sector publico, empresas y trabajadores) se prepare y gobierne el cambio de manera activa, con un amplio conjunto de políticas que garanticen la igualdad de oportunidades, potencien a largo plazo los efectos positivos de un progreso técnico y digital inclusivo, que ponga al alcance de todos las oportunidades de esta nueva era y reduzcan los costes de la transición a corto y medio plazo. Los principales retos de la economía española son:

- Facilitar un mercado de trabajo más eficiente y equitativo, con un crecimiento económico más inclusivo para reducir la desigualdad.

- Generar una mayor calidad institucional y apostar por la sostenibilidad y la eficiencia del sector público.

- Incentivar el aumento del tamaño de las empresas y potenciar una mayor internacionalización y competitividad.

- Promover la innovación, la adquisición y uso del capital tecnológico que promueva el incremento de la productividad y la competitividad de nuestra economía. 
En definitiva, eficiencia, equidad, sostenibilidad y competitividad global para mantener un sistema flexible ante las necesidades de un contexto «glocal» y VUCA, y justo para promover el estado del bienestar.

Algunas de las propuestas que pretenden dar respuesta al contexto actual podrían ser:

- Garantizar una renta mínima universal.

- Ordenar y regular las actividades en la economía colaborativa.

- Fiscalizar sobre las máquinas.

- Anticipar necesidades concretas de cualificaciones, por país, sector, región, e identificar los casos en los que una cooperación entre el sector educativo y el mundo empresarial han generado valor a la sociedad.

- Potenciar la formación a lo largo de la vida.

- Realizar análisis más rigurosos de la evolución y el desarrollo de las nuevas formas de trabajo.

- Ofrecer apoyo público más eficiente en las transiciones de un empleo a otro.

- Recomendar el diálogo social y las relaciones laborales.

- Reforzar el valor de las organizaciones empresariales y sindicales de manera que ofrezcan más servicios a sus afiliados.

- Promover una normativa laboral más predecible en Europa (seguridad jurídica), que contenga nuevos riesgos (responsabilidad de diferentes actores).

- Analizar la aparición de nuevas formas de agrupación colectiva y promover la defensa de los derechos de las empresas y las personas consumidoras que participan en estas nuevas formas de oferta y consumo colaborativo.

\section{Conclusiones}

En el último decenio se constata una tendencia generalizada del estado del bienestar (welfare) hacia un modelo caracterizado por ideas como la empleabilidad y la activación del mercado laboral (workfare). Este deslizamiento hacia el paradigma del workfare se acompaña de un cambio profundo en la perspectiva sobre la pobreza, abordada como «pobreza descalificadora» (Paugam, 2007), y también de un reforzamiento del discurso sobre la responsabilidad individual o «internalizada» (Schmidtz y Goodin, 2000).

En cualquier caso, lo que todas estas transformaciones socioeconómicas ponen de manifiesto es un desplazamiento desde el modelo de política social institucional redistributiva hacia un modelo de política social basado en el logro personal / resultado laboral, que apunta a un modelo residual de política social del bienestar. En este sentido, depende de los actores económicos que la transición de un mo- 
delo a otro, en un marco de adaptación a la complejidad del entorno, se realice de modo sostenible, justo e inclusivo.

\section{Referencias bibliográficas}

CARNOY, M.; CASTELLS, M. (1997). Sustainable flexibility: A prospective study on work, family and society in the information age. París: OECD.

DOMÉNECH, R. (2007). «Perspectivas macroeconómicas y retos del mercado de trabajo». Universidad Internacional Menéndez Pelayo (UIMP). Santander, 10 de julio.

EUROFOUND (2015). «New forms of employment». Luxemburgo: Publications Office of the European Union.

GALLINO, L. (2002). «La informalización del trabajo en los países desarrollados: cómo y por qué las condiciones de trabajo en el Norte se están aproximando a las del Sur». Sociología del Trabajo. N. ${ }^{\circ} 45$, págs. 7-24.

GORZ, A. (1995). Metamorfosis del trabajo. Crítica de la razón económica. Madrid: Sistema.

GUTIÉRREZ, R. (2017). «Viejas y nuevas ocupaciones». Encuentro CES «El futuro del trabajo». Universidad Complutense de Madrid, 17 de julio.

MUFFELS, T. J. A.; WILTHAGEN, T. (2006). "Conceptualization and Measurement of Flexicutiry in a Comparative Perspective». Seminar Flexicurity Network. Copenhague, 9 de junio.

OBSERVATORIO ADEI. «Notas técnicas». <http://observatorioadei.es/>

ONTIVEROS, I. (2015). «Digitalitzación industrial en España». El País Negocios, 20 de diciembre.

PAUGAM, S. (2007). Le salarié de la précarité: Les nouvelles formis de l'intégration professionnelle. Préface inédite de l'auteur. París: Presses universitaires de France.

SCHMIDTZ, D.; GOODIN, R. I. (2000). El bienestar social y la responsabilidad individual. Madrid: Ediciones Akal.

SUÁREZ, R. (2017). «Entendiendo el futuro del trabajo». Encuentro CES «El futuro del trabajo». Universidad Complutense de Madrid, 17 de julio. 


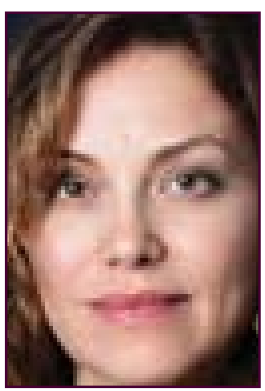

\section{Purificación Baldoví} baldoviborraspurificacion@gmail.com Profesora colaboradora de los Estudios de Economía y Empresa (UOC)

Economista por la Universidad de Valencia, licenciada en Ciencias del Trabajo por la UOC y máster en Recurso Humanos (ESIC), Innovación (UV) y Consejos de Dirección (UEV). Doctoranda en Innovación (UV). Vocal del Comité Económico y Social de la Comunidad Valenciana. En la actualidad, imparte talleres y ejerce como consultora en procesos de innovación empresariales, educativos y asociativos relacionados con el liderazgo, la negociación, la motivación y la gestión del equipo. Es también autora de contenidos docentes y profesora colaboradora de la UOC. En los últimos diez años ha sido la gerente de la Asociación Valenciana de Empresas Innovadoras en la Universidad Politécnica de Valencia y de la Fundación Globalis, dedicada a la innovación social dentro del contexto de la red nacional Innpulso.

Los textos publicados en esta revista están sujetas -salvo que se indique el contrario- a una licencia de Reconocimiento 3.0 España de Creative Commons. Podéis copiarlos, distribuirlos, comunicarlos públicamente y hacer obras derivadas siempre que reconozcáis los créditos de las obras (autoría, nombre de la revista, institución editora) de la manera especificada por los autores o por la revista. La licencia completa se puede consultar en http://creativecommons.org/licenses/by/3.0/es/deed.ca.

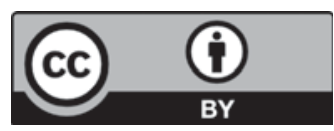

